\title{
Síntese de zeólitas a partir de cinza volante de caldeiras: caracterização física, química e mineralógica
}

\section{(Synthesis of zeolites from boiler fly ash: physical, chemical and mineralogical characterization)}

\author{
C. A. F. Rocha Junior', S. C. A. Santos' ${ }^{2}$ C. A. G. Souza', R. S. Angélica ${ }^{2}$, R. F. Neves ${ }^{1,2}$ \\ ${ }^{I}$ Programa de Pós Graduação em Engenharia Química (PPEQ-UFPA) \\ ${ }^{2}$ Programa de Pós-Graduação em Geologia e Geoquímica, Instituto de Geociências (PPGG-IG-UFPA), \\ Loteamento Cristo Redentor, R. Padre José de Anchieta 450A, Coqueiro, Ananindeua, PA 67125-165 \\ augustorocha2@gmail.com
}

\begin{abstract}
Resumo
Ao longo dos anos o desenvolvimento industrial trouxe uma crescente geração de resíduos, que são potenciais problemas ambientais Estudos das características destes materiais, bem como o desenvolvimento de técnicas para sua utilização se tornam imprescindíveis para a preservação do meio ambiente. Assim este trabalho objetiva a caracterização e utilização de cinza volante proveniente da queima de carvão mineral em caldeiras, assim como o uso de microssílica, utilizada para alterar a relação molar Si/Al da mistura cinza/microssílica na síntese de zeólitas. Para a caracterização da cinza foram utilizados métodos de análise química, física e mineralógica como: difração de raios X, fluorescência de raios X, microscopia eletrônica de varredura, análise granulométrica, análise térmica diferencial e termogravimétrica. Os ensaios de síntese foram feitos a $60,100,150$ e $190{ }^{\circ} \mathrm{C}$, com razão molar de $\mathrm{Na}_{2} \mathrm{O} / \mathrm{Al}_{2} \mathrm{O}_{3}$ igual a 5 e Si/Al variando de 2,12 a 15, e tempo de reação $24 \mathrm{~h}$. Os resultados da caracterização da cinza mostram potencial utilização deste como matéria-prima para a síntese de zeólitas, pois apresenta $\sim 50 \%$ de $\mathrm{SiO}_{2}$ e $\mathrm{Al}_{2} \mathrm{O}_{3}$, fases mineralógicas bem definidas, baixo teor de umidade, baixa granulometria $\left(\mathrm{d}_{90}<10 \mu \mathrm{m}\right)$, entre outros. Análises de difração de raios $\mathrm{X}$ dos produtos das sínteses mostraram a formação de alguns tipos de zeólitas, como analcima, phillipsita, sodalita, zeolita P e tobermorita. Deste modo, a mistura cinza e microssílica nestas condições reacionais mostram-se matérias-primas promissoras para a síntese de zeólitas. Palavras-chave: zeólita, síntese, cinza volante, meio ambiente.
\end{abstract}

\begin{abstract}
Along the years, worldwide industrial development has causing a growing generation of residues, bringing potentials environmental problems. A study of the characteristics of these wastes, as well as the development of techniques for their use in new processes becomes indispensable for the environment preservation. The main purpose of this work is to evaluate the possible use of two important industrial residues from the Amazon region for zeolite synthesis: (a) the fly ash (particle size $<100 \mu \mathrm{m})$ that comes from burning of mineral coal in boiler; and (b) the microsilica, a by-product of the reaction between quartz and coal in the production of metallic silicon and alloys iron-silicon. The following chemical, physical and mineralogical characterization methods were carried out: X-ray diffractometry, X-ray fluorescence, scanning electron microscopy, granulometric analysis, differential thermal and thermogravimetric analysis (DTA-TG). The analyses were carried out at the following conditions: 60, 100, 150 and $190{ }^{\circ} \mathrm{C}, \mathrm{Na} \mathrm{O}_{2} \mathrm{Al}_{2} \mathrm{O}_{3}$ molar ratio of 5 and $\mathrm{Si} / \mathrm{Al}$ molar ratio ranging from 2.12 to 15 , and reaction time of $24 \mathrm{~h}$. The results of the fly characterization demonstrate its enormous potential as raw material for the zeolite synthesis. $\mathrm{SiO}_{2}$ and $\mathrm{Al}_{2} \mathrm{O}_{3}$ represent more than $50 \%$ of its composition, mineralogical phases defined, low humidity content, low particle size $\left(d_{90}<10 \mu m\right)$, among others. Mineralogical analyses of the synthesized products showed the formation of some zeolite types, as follow: analcime, phillipsite, sodalite, zeolite $P$ and tobermorite. The results show that the mixture fly ash-microsilica in these reaction conditions point to a promising material for zeolite synthesis.
\end{abstract}

Keywords: zeolite, synthesis, fly ash, environment.

\section{INTRODUÇÃO}

O homem ao longo dos anos vem desenvolvendo uma série de processos para transformar resíduos industriais em materiais úteis à sociedade, diminuindo assim o seu impacto ambiental, preservando desta forma o meio ambiente. Um destes resíduos é a cinza de carvão mineral, gerada a partir da queima deste carvão em termoelétricas para a produção de vapor e/ou energia. Sua produção no âmbito mundial ultrapassa 550 milhões de ton/ano, sendo no contexto nacional gerado mais de 1,7 milhões de ton/ano, estando $80 \%$ relacionada a produção de cinza leve ou volante e $20 \%$ de cinza pesada ou de fundo $[1,2]$. Apesar desta grande produção, menos de $30 \%$ deste total é reutilizado, sobretudo para a produção de clínquer, utilizado na fabricação do cimento Portland, ficando o restante disposto em bacias 
de rejeito ou utilizado para recompor minas de carvão desativadas [3-9]. Como é previsto um aumento de mais de $100 \%$ no consumo de carvão para fins termoelétricos no Brasil, a geração de cinzas deverá chegar a 3,5 ou a 4 milhões de ton/ano, enquanto que o seu uso pela indústria cimenteira não terá certamente aumento significativo para compensar este crescimento [2,10]. Assim, processos que transformem esta cinza em material de alto valor agregado tornam-se necessários, a fim de diminuir a sua presença no meio ambiente. A semelhança na composição química da cinza de carvão, com o material vulcânico precursor das zeólitas naturais foi a principal razão do desenvolvimento de experimentos envolvendo a síntese de zeólitas a partir desta matéria-prima $[11,12]$. Embora o potencial de aplicação do produto sintetizado possa consumir somente uma pequena porção das cinzas geradas em termelétricas, os produtos finais obtidos alcançam um maior valor agregado do que aquele obtido na construção civil [13, 14], o que estimula ainda mais a sua utilização neste processo.

Zeólitas são aluminossílicatos hidratados cristalinos com elementos do grupo I e II da tabela periódica. Sua estrutura está baseada em infinitas redes tridimensionais de tetraedros de $\left[\mathrm{SiO}_{4}\right]^{4-}$ e $\left[\mathrm{AlO}_{4}\right]^{5-}$ unidos pelos vértices por oxigênio. Estas redes formam uma grande quantidade de espaços vazios e abertos, sendo estas responsáveis pela definição de inúmeras propriedades especiais das zeólitas. A substituição de $\mathrm{Si}^{4+}$ por $\mathrm{Al}^{3+}$ nos tetraedros explica a carga negativa da estrutura, o que gera uma alta capacidade de troca iônica quando os poros e canais permitem o acesso dos cátions $[15,16]$. Como conseqüência desta peculiar estrutura das zeólitas, estas podem ser usadas em uma ampla faixa de aplicações industriais como material para troca iônica, adsorvente, catalisador, dentre outras, todas baseadas em sua seletividade eletrônica [16].

O uso da cinza como material zeolítico vem sendo estudado há vários anos, sendo H. Höller e U. Wirsching os pioneiros, tendo obtido bons resultados em sua utilização [17]. Posteriormente, várias patentes e artigos científicos foram produzidos sugerindo diversos métodos de síntese a partir deste material, para obtenção de diversos tipos de zeólitas [18-23]. A síntese alcalina a partir de cinza ocorre em três etapas: dissolução, formação de gel e cristalização [5]. A dissolução da cinza ocorre entre 25 e $120^{\circ} \mathrm{C}$, sendo muito importante a concentração de hidróxido nesta etapa. Posteriormente há uma reação com a formação de um gel a partir de íons de alumínio e silício na superfície das partículas da cinza, ficando as concentrações destes metais no meio reacional, limitadas pelo progresso desta reação. A cristalização da zeólita ocorre a partir do aluminossilicato formado, sendo a taxa de cristalização controlada pela concentração do hidróxido usado no processo [3,5].

As características físicas, químicas e mineralógicas das cinzas volantes dependem de uma variedade de fatores: a composição do carvão que lhes deu origem, as condições de combustão, tipo e eficiência do sistema de controle de emissões e os métodos de disposição utilizados. Conseqüentemente, é difícil generalizar sobre suas características, o que torna necessário um estudo prévio a cerca deste material, principalmente para fins zeolíticos, uma vez que a síntese de zeólitas é afetada diretamente pelas características do seu material precursor [4, 9, 24]. Dessa forma este trabalho tem como objetivo a caracterização de cinzas volantes provenientes de uma planta de alumina da região norte do Brasil, a fim de avaliar o seu potencial como matéria-prima (fonte de sílica e alumina) no processo de síntese de zeólitas, bem como utilizar microssílica $\left(\mathrm{SiO}_{2}\right)$, neste processo para que haja uma variação na relação molar de $\mathrm{Si} / \mathrm{Al}$, buscando a maximização dos diferentes tipos de zeólitas.

\section{MATERIAIS E MÉTODOS}

Difração de raios $X(D R X)$ : as análises foram feitas em difratômetro X'Pert PRO MPD (PW 3040/60) PANalytical, com goniômetro PW3050/60 (teta/teta), tubo cerâmico de anodo de $\mathrm{Cu}\left(\mathrm{k} \alpha_{1} 1,540598 \AA\right.$ A $)$ PW3373/00, com foco fino longo, filtro $\mathrm{k} \beta$ de $\mathrm{Ni}$, potência $2200 \mathrm{~W}, 60 \mathrm{kV}$ e com detector do tipo RTMS X'Celerator. A aquisição de dados foi feita com o software X'Pert Data Collector, versão 2.1a, e o tratamento dos dados com o software X'Pert HighScore versão $2.1 b$.

Espectrometria de fluorescência de raios X (FRX): a composição química da cinza volante e microssílica foi obtida por FRX em um espectrômetro seqüencial Axios Minerals PANalytical, sendo a aquisição dos dados feita com o software SuperQ Manager e o tratamento com o software IQ+ Semiquant. Na análise de FRX da cinza foi feita uma pastilha prensada com cerca de $3 \mathrm{~cm}$ de diâmetro, uma mistura de $3 \mathrm{~g}$ da cinza e $0,6 \mathrm{~g}$ de parafina (utilizada como aglomerante). Já para a análise da microssílica foi feito um disco de vidro com aproximadamente $1 \mathrm{~g}$ de amostra e $8 \mathrm{~g}$ de tetraborato de lítio $\left(\mathrm{Li}_{2} \mathrm{~B}_{4} \mathrm{O}_{7}\right)$.

Teste de lixiviação: $\mathrm{O}$ teste de lixiviação na cinza volante foi realizado conforme a norma ABNT [25]. Para este teste pesou-se $100 \mathrm{~g}$ da amostra, sendo posteriormente transferido para um frasco de politetrafluoretileno, no qual foram adicionados $2000 \mathrm{~g}$ de solução de extração de ácido acético com pH 2,91. Esta solução foi escolhida a partir de testes estabelecidos também pela norma ABNT NBR 10005:2004. O frasco contendo a cinza volante, bem como a solução extratora, foi agitado por $20 \mathrm{~h}$. Em seguida, a mistura foi filtrada a vácuo com membrana filtrante de porosidade entre 0,6 e $0,8 \mu \mathrm{m}$. A concentração dos metais pesados no extrato lixiviado foi determinada a partir da diferença entre a concentração destes na cinza volante in natura e a concentração na cinza lixiviada, por meio da análise química por FRX.

Microscopia eletrônica de varredura (MEV): as análises da cinza volante e dos produtos das sínteses foram feitas em um microscópio Leo 1430. Posteriormente as amostras foram montadas em suportes de alumínio com $10 \mathrm{~mm}$ de diâmetro com fita adesiva de carbono, sendo feita a metalização com uma película de platina de espessura de $\sim 15 \mathrm{~nm}$, em equipamento Emitech K550, a uma pressão de $2.10^{-1} \mathrm{mbar}$, 
e corrente de $25 \mathrm{~mA}$ durante $2,5 \mathrm{~min}$. As imagens foram geradas por elétrons secundários com voltagens de $20 \mathrm{kV}$.

Análise granulométrica: foi utilizado o equipamento laser particle sizer analysette 22 Fritsch com software MaScontrol para a aquisição dos dados.

Análise térmica diferencial e termogravimétrica (ATD e $A T G$ ): as análises da cinza volante foram feitas em um equipamento Thermal Sciences PL, com analisador térmico simultâneo STA 1000/1500 Stanton Redcroft Ltd., em forno cilindro vertical, conversor digital acoplado a um microcomputador, termopar de Pt-Rh e cadinho de alumina com $\sim 10 \mathrm{mg}$ de amostra da temperatura ambiente até $1100{ }^{\circ} \mathrm{C}$, com taxa de aquecimento de $10{ }^{\circ} \mathrm{C} / \mathrm{min}$.

Processo de síntese: o principal material de partida é a cinza volante, oriunda de uma produtora de alumina da região norte do Brasil, sendo a principal fonte de Si e Al para a síntese de zeólitas. Adicionalmente, foi utilizada uma microssílica comercial, como fonte de $\mathrm{Si}$, além de hidróxido de sódio micropérolas PA (Vetec Química Fina) como fonte de metal alcalino. Foi feita uma calcinação prévia da cinza a $650{ }^{\circ} \mathrm{C}$ por $2 \mathrm{~h}$ para a eliminação de resíduos de carbono, em decorrência da queima incompleta do carvão mineral. Posteriormente, o produto da calcinação foi homogeneizado e alíquotas foram retiradas e pesadas para as etapas seguintes. Como as análises químicas preliminares da cinza revelaram uma razão molar de $\mathrm{Si} / \mathrm{Al}$ igual a 2,12, relativamente baixa para a síntese de vários tipos de zeólitas, optou-se pela utilização de uma fonte adicional de silício (microssílica), a fim de maximizar a variedade das zeólitas, bem como investigar a melhor relação molar de $\mathrm{Si} / \mathrm{Al}$ para a síntese a partir da cinza volante, como mostra a Tabela I.

Tabela I - Massa de microssílica utilizada para cada relação molar de Si/Al no processo de síntese.

[Table I - Microsilica mass used for each Si/Al molar ratio in the synthesis process.]

\begin{tabular}{cc}
\hline $\begin{array}{c}\text { Relação molar } \\
\mathrm{Si} / \mathrm{Al}\end{array}$ & $\begin{array}{c}\text { Massa de } \\
\text { microssílica }(\mathrm{g})\end{array}$ \\
\hline 2,12 & 0 \\
3 & 0,1440 \\
5 & 0,4751 \\
7 & 0,8061 \\
9 & 1,1371 \\
11 & 1,4698 \\
13 & 1,8025 \\
15 & 2,1368 \\
\hline
\end{tabular}

A metodologia de síntese consistiu na adição de $1 \mathrm{~g}$ de cinza volante em 8 reatores com volume $50 \mathrm{~mL}$, bem como a adição de $30 \mathrm{~mL}$ de água, $10,2 \mathrm{~mL}$ de $\mathrm{NaOH}$ a $5 \mathrm{~N}$ e a quantidade de microssílica determinada para cada razão molar de Si/Al (Tabela I). Posteriormente, os reatores foram fechados e colocados em uma estufa de laboratório sem recirculação de ar, a $60,100,150$ e $190{ }^{\circ} \mathrm{C}$ por 24 h. Em seguida, os reatores foram retirados da estufa e deixados esfriar até a temperatura ambiente, sendo filtrado e lavado com $700 \mathrm{~mL}$ de água deionizada todo o produto reacional dos reatores. $\mathrm{O}$ material retido nos filtros foi seco em estufa a $110{ }^{\circ} \mathrm{C}$ por $3 \mathrm{~h}$, sendo posteriormente transferido para um dessecador até o seu resfriamento, sendo em seguida pesado e acondicionado em frascos de polietileno para posterior análise mineralógica.

\section{RESULTADOS E DISCUSSÃO}

\section{Caracterização da cinza volante}

A caracterização química da cinza volante é bastante importante tanto com relação aos elementos majoritários quanto aos elementos traços presentes em sua composição, pois estes influenciam fortemente no seu potencial de aplicação, bem como determinam seu impacto no meio ambiente. Os resultados da análise química por espectrometria de fluorescência de raios $\mathrm{X}$ da cinza volante estão apresentados na Tabela II. Quase $50 \%$ da composição deste material apresenta $\mathrm{SiO}_{2}(33,72 \%)$ e $\mathrm{Al}_{2} \mathrm{O}_{3}(13,32 \%)$ como óxidos principais, sendo esta característica esperada uma vez que estes são os principais constituintes das cinzas de carvão mineral $[6,8,24,26,27]$. Estes altos teores de $\mathrm{SiO}_{2}$ e $\mathrm{Al}_{2} \mathrm{O}_{3}$ encontrados na cinza conferem a esta uma grande aplicação como matéria-prima na síntese de zeólitas, uma vez que estes óxidos são um dos componentes básicos das estruturas zeolíticas [3, 26, 28]. Outra importante característica a se avaliar a partir da análise química da cinza volante para fins zeolíticos é a presença de algumas impurezas, como por exemplo $\mathrm{Fe}_{2} \mathrm{O}_{3}$ e $\mathrm{CaO}$, que atuam de forma negativa durante a formação deste material. $\mathrm{O}$ óxido de ferro é responsável pela diminuição da cristalização das zeólitas e dissolução do silício e alumínio no meio reacional, uma vez que este ocupa preferencialmente a superfície das

Tabela II - Resultado da análise química por espectrometria de fluorescência de raios $\mathrm{X}$ da cinza volante.

[Table II - XRF results of the fly ash sample.]

\begin{tabular}{cccc}
\hline Composto & $\begin{array}{c}\text { Concentração } \\
(\%)\end{array}$ & $\begin{array}{c}\text { Elementos } \\
\text { traços }\end{array}$ & $\begin{array}{c}\text { Concentração } \\
\text { (ppm) }\end{array}$ \\
\hline $\mathrm{Na}_{2} \mathrm{O}$ & 0,76 & $\mathrm{As}$ & 163 \\
$\mathrm{MgO}$ & 1,49 & $\mathrm{Ba}$ & 56 \\
$\mathrm{Al}_{2} \mathrm{O}_{3}$ & 13,32 & $\mathrm{~Pb}$ & 140 \\
$\mathrm{SiO}_{2}$ & 33,72 & $\mathrm{Cr}$ & 120 \\
$\mathrm{SO}_{3}$ & 6,48 & $\mathrm{~V}$ & 206 \\
$\mathrm{~K}_{2} \mathrm{O}$ & 1,31 & $\mathrm{Co}$ & 19 \\
$\mathrm{CaO}$ & 14,44 & $\mathrm{Ni}$ & 75 \\
$\mathrm{TiO}_{2}$ & 0,73 & $\mathrm{Cu}$ & 55 \\
$\mathrm{Fe}_{2} \mathrm{O}_{3}$ & 5,75 & $\mathrm{Zn}$ & 75 \\
$\mathrm{PF}^{*}$ & 22,20 & $\mathrm{Sr}$ & 3 \\
$\mathrm{Total}^{*}$ & 100,20 & $\mathrm{Mn}$ & 492 \\
\hline
\end{tabular}

*Perda ao Fogo 
partículas da cinza, dificultando processo difusivo entre estas e o meio $[3,16]$. $\mathrm{CaO}$ assim como $\mathrm{Fe}_{2} \mathrm{O}_{3}$ diminui o rendimento do processo de síntese de zeólitas, porém o seu efeito é bem mais expressivo para zeólitas do tipo A onde teores acima de 4,5\% inibem a sua formação [2, 16]. Observando-se estes dois teores a partir da Tabela II, podemos verificar valores bastante significativos, 5,75\% para o $\mathrm{Fe}_{2} \mathrm{O}_{3}$ e $14,44 \%$ para o $\mathrm{CaO}$, que podem dificultar a formação das zeólitas.

Outro aspecto relacionado às concentrações de $\mathrm{SiO}_{2}$, $\mathrm{Al}_{2} \mathrm{O}_{3}$ e $\mathrm{Fe}_{2} \mathrm{O}_{3}$ está no fato de que a soma destes óxidos podem classificar a cinza volante como classe $\mathrm{C}$, uma vez que apresentam a soma de suas concentrações maiores que $50 \%$, evidenciando propriedades cimentantes e pozolânicas [29]. O valor de perda ao fogo (PF) para a cinza volante mostra-se muito expressivo $(22,20 \%)$ sendo a maior parte relacionada à perda da matéria orgânica, como será descrito a seguir nos resultados das análises térmicas, bem como a perda de $\mathrm{CO}_{2}$ da estrutura da calcita e de $\mathrm{SO}_{4}$, da estrutura da anidrita. As concentrações dos metais pesados presentes na cinza volante in natura, cinza lixiviada e extrato lixiviado, bem como os limites estabelecidos pela norma ABNT NBR 10004:2004 para estes metais são mostrados na Tabela III. Todos os limites estabelecidos pela norma para a concentração do $\mathrm{As}, \mathrm{Ba}, \mathrm{Pb}$ e Cr no extrato lixiviado estão acima do valor permitido [30], o que caracteriza a cinza volante utilizada neste trabalho como resíduo de classe I, ou seja perigoso. Esta característica é comumente encontrada em outros trabalhos com cinza volante [31-33]. O alto valor no $\mathrm{pH}$ igual a 12,49 da cinza in natura está intimamente ligado à alta concentração de $\mathrm{CaO}(14,44 \%)$ neste material, relacionado principalmente à presença de cálcio sob a forma de $\mathrm{CaO}$ amorfo e anidrita $\left(\mathrm{CaSO}_{4}\right)$. Apesar dos altos teores de metais pesados encontrados no extrato lixiviado, estes não se configuram como grande problema durante a síntese de zeólitas, uma vez que o material zeolítico imobilizará grande parte destes metais [34], evitando assim contaminações durante a sua aplicação, assim como a solução remanescente do processo de zeolitização poderá ser tratada para posterior descarte no meio ambiente.

Tabela III - Concentração dos metais pesados na cinza volante in natura, cinza lixiviada e extrato lixiviado.

[Table III - Concentration of the heavy metals in the nature fly ash, leached fly ash and leached extract.]

\begin{tabular}{ccccc}
\hline & $\begin{array}{c}\text { Limite } \\
\text { máximo } \\
\text { ABNT NBR } \\
\text { 10004:2004 } \\
(\mathrm{ppm})\end{array}$ & $\begin{array}{c}\text { Cinza volante } \\
\text { in natura } \\
\text { (ppm) }\end{array}$ & $\begin{array}{c}\text { Cinza } \\
\text { lixiviada } \\
\text { (ppm) }\end{array}$ & $\begin{array}{c}\text { Extrato } \\
\text { lixiviado } \\
\text { (ppm) }\end{array}$ \\
\hline $\mathrm{pH}$ & - & 12,49 & 6,14 & 6,14 \\
$\mathrm{As}$ & 1 & 163 & 144 & 19 \\
$\mathrm{Ba}$ & 70 & 562 & 435 & 127 \\
$\mathrm{~Pb}$ & 1 & 140 & 134 & 6 \\
$\mathrm{Cr}$ & 5 & 120 & 93 & 27 \\
\hline
\end{tabular}

Analisando o difratograma da Fig. 1 observa-se o quartzo como fase mineralógica principal da cinza volante, bem como se identifica a presença de outras, como albita $\left(\mathrm{AlNaO} \mathrm{Si}_{3}\right)$, hematita $\left(\mathrm{Fe}_{2} \mathrm{O}_{3}\right)$, anidrita $\left(\mathrm{CaSO}_{4}\right)$, calcita $\left(\mathrm{CaCO}_{3}\right)$ e muscovita $\left(\mathrm{KAl}_{2} \mathrm{Si}_{3} \mathrm{AlO}_{10}(\mathrm{OH})_{2}\right)$, sendo estas freqüentemente encontradas em cinzas de carvão mineral [8, 24]. A presença de anidrita na cinza volante está intimamente ligada ao processo de dessulfuração interna utilizado durante a queima do carvão mineral, onde há a inserção de calcário $\left(\mathrm{CaCO}_{3}\right)$ ao longo desta queima, a fim de diminuir as emissões de gases $\mathrm{SO}_{\mathrm{x}}$ para a atmosfera, conforme as reações apresentadas nas equações A e B.

$$
\begin{aligned}
& \mathrm{CaCO}_{3} \rightarrow \mathrm{CaO}+\mathrm{CO}_{2} \\
& \mathrm{CaO}+\mathrm{SO}_{2}+1 / 2 \mathrm{O}_{2} \rightarrow \mathrm{CaSO}_{4}
\end{aligned}
$$

Algumas fases identificadas na cinza volante estão relacionadas a temperatura utilizada durante a combustão do carvão, sendo que para processos a temperaturas inferiores a $1000{ }^{\circ} \mathrm{C}$, algumas fases cristalinas ou amorfas presentes originalmente no carvão se mantêm durante todo o processo, uma vez que estas temperaturas não são suficientes para destruir essas estruturas, como por exemplo, o quartzo, hematita, albita e muscovita $[2,26]$. Tal fato justifica a presença destes minerais no difratograma (Fig. 1), em decorrência da cinza volante utilizada neste trabalho ser produzida a $\sim 800{ }^{\circ} \mathrm{C}$. Outro ponto a se observar na Fig. 1 é o aumento do background do difratograma entre 20 a $32^{\circ}(2 \theta)$. Este se justifica pela presença de fases amorfas, principalmente de carbono incombusto (residual) e fases vítreas de aluminossilicato. Estas fases vítreas são responsáveis por grande parte do teor de alumínio determinado pela análise química, visto que a muscovita identificada na difração de raios $\mathrm{X}$ não seria capaz de

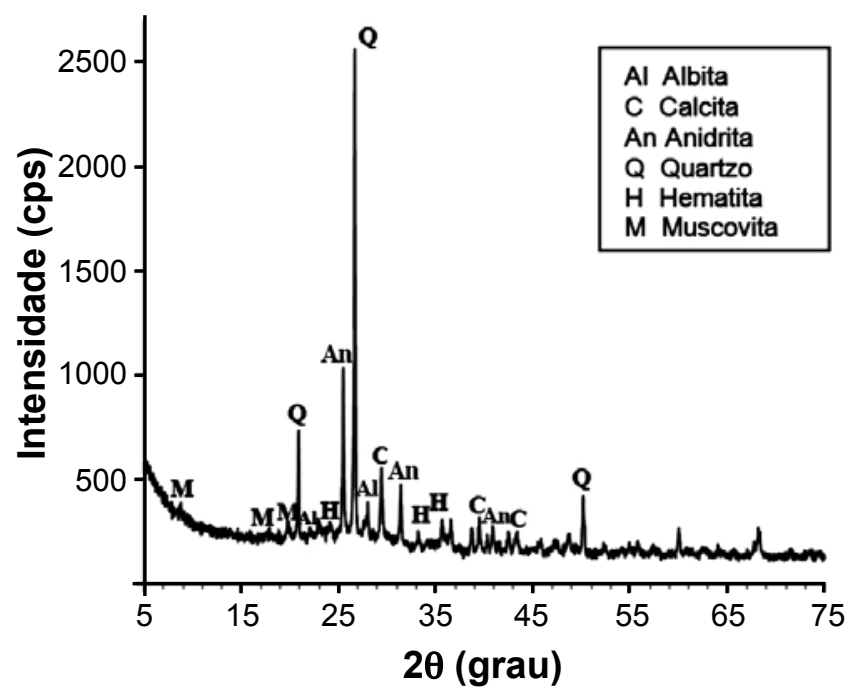

Figura 1: Difratograma de raios $\mathrm{X}$ da cinza volante apresentando 5 fases mineralógicas com seus 3 principais picos.

[Figure 1: XRD pattern of the fly ash showing 5 mineralogical phases with their 3 main peaks.] 
apresentar tal teor, uma vez que as intensidades de seus picos refletem uma baixa concentração desta fase.

As micrografias da Fig. 2 revelam partículas de tamanhos e formas bastante variadas, relacionados aos minerais já descritos por DRX. A Fig. 2a apresenta partículas de carbono residual $(\mathrm{Ca})$ do processo de combustão do carvão mineral, já na Fig. 2b observam-se grãos de quartzo (Q) bem euédricos. Nas Figs. 2c e $2 \mathrm{f}$ podem ser observados cristais prismáticos ortorrômbicos de anidrita (An), evidenciando um caráter de neoformação a partir do processo de dessulfuração como já apresentado. Cristais fibro radiais de muscovita (M) podem ser observados na Fig. 2d, enquanto que nas Figs. 2e e 2f são nítidos os cristais romboédricos de calcita (C).

Analisando a curva termogravimétrica apresentada na Fig. 3, podemos observar que não existe variação significativa na massa da cinza volante até aproximadamente $400{ }^{\circ} \mathrm{C}$, o que demonstra que não há umidade superficial nesta amostra, bem como a presença de material orgânico
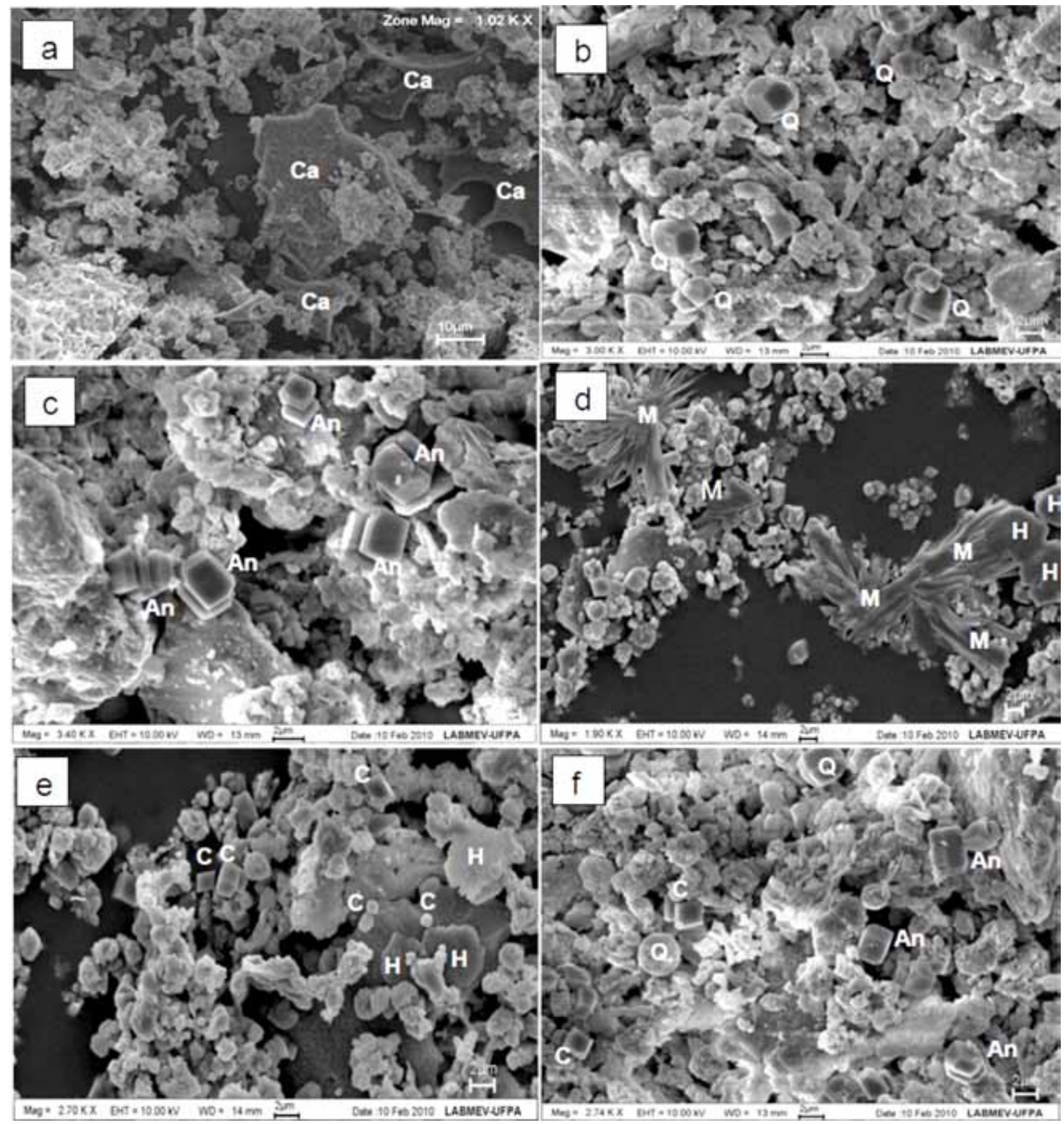

Figura 2: Micrografias obtidas por microscopia eletrônica de varredura mostrando diferentes morfologias das fases mineralógicas encontradas na cinza volante: a) partículas de carbono residual; b) quartzo euédrico; c) cristais ortorrômbicos de anidrita; d) hematita e muscovita; e) romboedros de calcita e hematita; f) anidrita, calcita e quartzo.

[Figure 2: SEM micrographs showing different morphologies found in the fly ash: a) particles of residual carbon; b) quartz; c) anhydrite; d) hematite and muscovite; e) calcite and hematite; f) anhydrite, calcite and quartz.] 
volátil que se decompõem até esta temperatura. Entre 400 e $674{ }^{\circ} \mathrm{C}$ nota-se um expressivo pico exotérmico (Ex) na curva térmica diferencial (ATD), sendo a combustão (C) do carbono residual, responsável pelo aparecimento deste pico. Tal fato indica falha no processo de queima do carvão mineral, uma vez que em condições normais de operação, os fornos modernos não produzem cinzas volantes contendo mais de 5\% de carbono [35]. Esta combustão ficou bastante evidente após a análise, pois observou-se uma grande mudança na coloração da amostra, passando de cinza escuro para marrom claro.

$$
\mathrm{C}+\mathrm{O}_{2} \rightarrow \mathrm{CO}_{2}
$$

$\mathrm{Na}$ análise térmica também se verificou a presença de um pico endotérmico (En) entre 674 a $774{ }^{\circ} \mathrm{C}$ aproximadamente, sendo este formado a partir da reação (D) de decomposição da calcita adicionada no processo de dessulfuração, como já apresentado.

$$
\mathrm{CaCO}_{3} \rightarrow \mathrm{CaO}+\mathrm{CO}_{2}
$$

As reações de combustão do carbono residual, bem como da decomposição da calcita são responsáveis por uma perda de massa de aproximadamente $20 \%$ da cinza volante, sendo $15 \%$ da combustão e $5 \%$ da decomposição, como podemos observar na curva termogravimétrica. Estes resultados corroboram a análise de perda ao fogo (PF) mostrado na Tabela II.

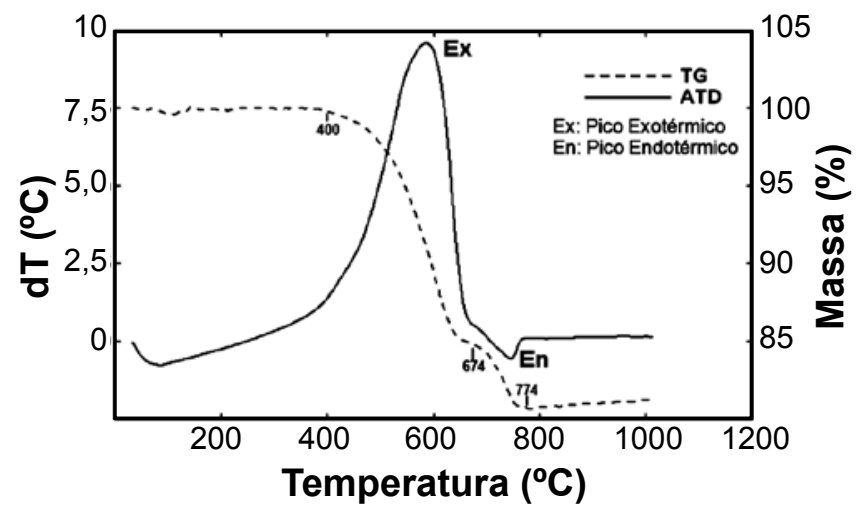

Figura 3: Gráfico da análise térmica da cinza volante.

[Figure 3: Thermal analysis of the fly ash sample.]

A Fig. 4 mostra que mais de $90 \%$ das partículas $\left(\mathrm{d}_{90}\right)$ da cinza volante apresentam granulometria abaixo de $10 \mu \mathrm{m}$, o que está de acordo tanto com a definição geral de cinza volante (granulometria entre $0,5 \mu \mathrm{m}$ e $100 \mu \mathrm{m}$ ), quanto com as principais normas de caracterização deste tipo de cinza [9]. Essas normas estabelecem um limite mínimo de quantidade de partículas retidas na peneira de 325 Tyler (44 $\mu \mathrm{m}$ de abertura de malha) como, por exemplo, a norma européia (NP EN 450) que estabelece que a percentagem de material retido não deve exceder $40 \%$, a americana (ASTM C 618) e a canadense que indicam o valor limite de $34 \%$, a australiana na qual o limite é de $50 \%$ e a japonesa $25 \%$ [14]. Esta distribuição granulométria da cinza volante é de grande importância durante o processo de síntese de zeólitas, pois alguns estudos mostram que tal característica aumenta a reatividade destas partículas e conseqüentemente aceleram o seu processo de zeolítização $[3,36]$.

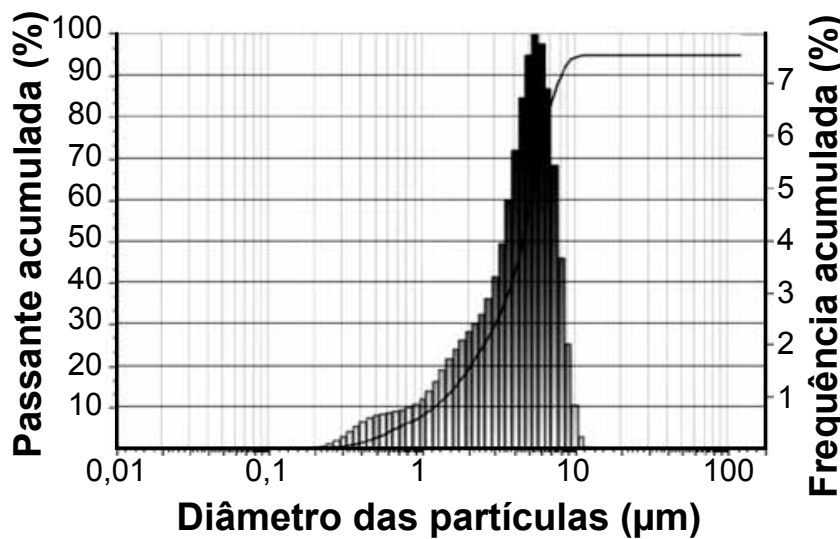

Figura 4: Distribuição granulométrica da cinza volante. [Figure 4: Granulometric analysis of the fly ash sample.]

Estudos mostram que os altos teores de fases não reativas durante o processo de síntese dificultam a zeolitização da matéria-prima, diminuindo assim o seu rendimento $[2,3$, $16,18,36]$. Isso justifica a calcinação da cinza em questão, em função da grande quantidade de carbono incombusto na amostra, como descrito anteriormente nos resultados das análises térmicas. A temperatura de calcinação utilizada foi de $650{ }^{\circ} \mathrm{C}$, pois de acordo com a curva térmica diferencial há uma completa combustão deste carbono até esta temperatura, bem como evitará a decomposição da calcita em óxido de cálcio, pois tal reação ocorre aproximadamente em $674{ }^{\circ} \mathrm{C}$. A análise de DRX da cinza após a calcinação (Fig. 5) não mostra novas fases mineralógicas, bem como a destruição das fases originais, porém houve uma sensível diminuição do background, entre aproximadamente 20 e $32^{\circ}$

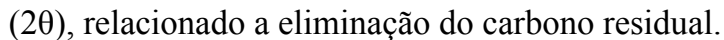

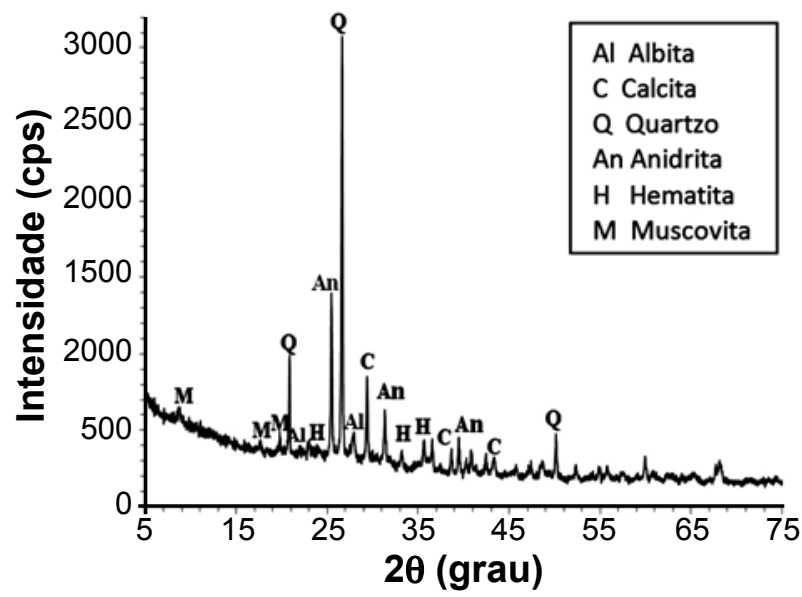

Figura 5: Difratograma de raios $\mathrm{X}$ da cinza volante calcinada. [Figure 5: XRD pattern of the calcined fly ash sample.] 
A análise química da microssílica comercial utilizada na síntese (Tabela IV), demonstra que este material apresenta um alto teor de $\mathrm{SiO}_{2}$, bem como baixo teor de impurezas, o que torna sua utilização bastante eficiente neste processo, pois a síntese de zeólitas é fortemente afetada por alguns parâmetros como teor de $\mathrm{Fe}$, metais alcalinos, umidade e outros [3].

A Tabela $V$ mostra os diferentes tipos de zeólitas obtidos a partir da mistura cinza/microssílica em função dos diferentes parâmetros reacionais adotados. Não houve formação de material zeolítico para as relações molares de $\mathrm{Si} / \mathrm{Al}$ a $60{ }^{\circ} \mathrm{C}$, porém trabalhos mostraram a formação de faujasita (zeólita $\mathrm{X}$ ) a partir destas condições reacionais [37, 38]. Análises de DRX destes produtos (Fig. 6a) revelam ainda uma grande intensidade nos picos de quartzo, mostrando a baixa solubilidade desta fase a estas condições reacionais. Tal fato influencia significativamente a formação das zeólitas, uma

Tabela IV - Análise de fluorescência de raios $\mathrm{X}$ da microssílica.

[Table IV - XRF analysis of the microsilica sample.]

\begin{tabular}{cc}
\hline Composto & Concentração (\%) \\
\hline $\mathrm{Na}_{2} \mathrm{O}$ & 0,19 \\
$\mathrm{MgO}$ & 0,3 \\
$\mathrm{Al}_{2} \mathrm{O}_{3}$ & 0,05 \\
$\mathrm{SiO}_{2}$ & 96,3 \\
$\mathrm{~K}_{2} \mathrm{O}$ & 0,65 \\
$\mathrm{CaO}$ & 0,28 \\
$\mathrm{Fe}_{2} \mathrm{O}_{3}$ & 0,05 \\
$\mathrm{PF}$ & 1,69 \\
Total & 99,32 \\
\hline
\end{tabular}

vez que a taxa de dissolução desta fase em conjunto com as que contêm $\mathrm{Al}$ determinam a relação molar de $\mathrm{Si} / \mathrm{Al}$ na fase liquida e por conseguinte o tipo de zeólita formada [2].

A partir de $100{ }^{\circ} \mathrm{C}$ observou-se a formação zeolítica para todas as relações molares de $\mathrm{Si} / \mathrm{Al}$, indicando que a temperatura foi uma variável fundamental para produção destas fases, uma vez que os outros parâmetros de processo foram mantidos constantes. Apesar da microssílica não ter exercido uma influência significativa no processo de síntese, por outro lado teve um papel fundamental na maximização dos tipos de zeólitas, uma vez que sem a utilização desta fonte extra de silício somente seria possível à formação de dois produtos zeolíticos, a sodalita e torbemorita, como podemos observar na relação molar de $\mathrm{Si} / \mathrm{Al}$ igual a 2,12 para todas as temperaturas. A maioria das zeólitas produzidas a partir da cinza volante foi formada em conjunto. Porém, observa-se a presença de uma única fase zeolítica em apenas três relações molares de $\mathrm{Si} / \mathrm{Al}: 2,12 ; 13$ e 15 à temperatura de $100{ }^{\circ} \mathrm{C}$, como podemos ver nas análises de DRX destes produtos (Figs. 6b, c e d).

Apesar de haver uma única fase zeolítica nestes produtos, os picos desta fase não apresentam grande intensidade, revelando menor concentração em comparação aos minerais presentes inicialmente na cinza (quartzo, hematita, albita, anidrita, calcita e muscovita). O domínio das fases zeolíticas sobre os minerais originais da cinza são observados somente para $150{ }^{\circ} \mathrm{C}$ e $190{ }^{\circ} \mathrm{C}$ para todas as relações molares de $\mathrm{Si} /$ $\mathrm{Al}$, a exemplo a relação igual a 7 sintetizada a $190^{\circ} \mathrm{C}$, como mostra a Fig. 7. Nesta, podemos observar a total dissolução do quartzo, que está relacionada à zeolítização da cinza volante [39]. Análises de microscopia eletrônica deste produto mostram cristais com morfologia trapezoédrica com raros octaedros de analcima, bem como cristais aciculares de pectolita (Fig. 8).

Tabela V - Produtos obtidos na síntese de zeólitas.

[Table V-Products obtained in the zeolite synthesis process.]

\begin{tabular}{|c|c|c|c|c|c|c|c|c|c|c|c|c|c|c|c|c|c|c|c|}
\hline \multicolumn{20}{|c|}{ Produtos da síntese } \\
\hline & \multicolumn{5}{|c|}{$60^{\circ} \mathrm{C}$} & \multicolumn{5}{|c|}{$100^{\circ} \mathrm{C}$} & \multicolumn{5}{|c|}{$150^{\circ} \mathrm{C}$} & \multicolumn{4}{|c|}{$190^{\circ} \mathrm{C}$} \\
\hline $\begin{array}{l}\text { Razão Molar } \\
\text { de Si/Al }\end{array}$ & A & $\mathrm{P}$ & $\mathrm{S}$ & $\mathrm{T}$ & $\mathrm{Zp}$ & $A$ & $\mathrm{P}$ & $\mathrm{S}$ & $\mathrm{T}$ & $\mathrm{Zp}$ & A & $\mathrm{P}$ & $\mathrm{S}$ & $\mathrm{T}$ & $\mathrm{Zp}$ & A & $\mathrm{P} S$ & $\mathrm{~T}$ & $\mathrm{Zp}$ \\
\hline $2,12^{*}$ & - & - & - & - & - & . & - & $\mathrm{X}$ & - & - & & - & $\mathrm{X}$ & $\mathrm{X}$ & - & - & $-X$ & $\mathrm{X}$ & - \\
\hline 3 & - & - & - & - & - & . & $\mathrm{X}$ & - & - & $\mathrm{X}$ & $\mathrm{X}$ & $\mathrm{X}$ & $\mathrm{X}$ & $\mathrm{X}$ & - & $X$ & $-X$ & $\mathrm{X}$ & - \\
\hline 5 & - & - & - & - & - & & $\mathrm{X}$ & - & - & $\mathrm{X}$ & $\mathrm{X}$ & $\mathrm{X}$ & - & - & $\mathrm{X}$ & $X$ & - - & $\mathrm{X}$ & - \\
\hline 7 & - & - & - & - & - & . & $\mathrm{X}$ & - & - & $\mathrm{X}$ & $\mathrm{X}$ & $\mathrm{X}$ & - & - & $\mathrm{X}$ & $X$ & - - & $\mathrm{X}$ & - \\
\hline 9 & - & - & - & - & - & . & $\mathrm{X}$ & - & - & $\mathrm{X}$ & $\mathrm{X}$ & $\mathrm{X}$ & - & - & $\mathrm{X}$ & $X$ & $-X$ & - & - \\
\hline 11 & - & - & - & - & - & . & $X$ & - & - & $X$ & $\mathrm{X}$ & $\mathrm{X}$ & - & - & $\mathrm{X}$ & $X$ & $-X$ & - & - \\
\hline 13 & - & - & - & - & - & . & - & - & - & $X$ & $X$ & $\mathrm{X}$ & - & - & $\mathrm{X}$ & $X$ & $-\quad-$ & - & X \\
\hline 15 & - & - & - & - & - & . & - & - & - & $\mathrm{X}$ & $\mathrm{X}$ & $\mathrm{X}$ & - & - & $\mathrm{X}$ & $X$ & $-X$ & - & - \\
\hline
\end{tabular}

(A) analcima, (P) phillipsta, (S) sodalita, (T) tobermorita e (Zp) zeólita P. (X) Zeólita sintetizada, (-) Zeólita não formada. * Não houve adição de microssílica neste produto. 

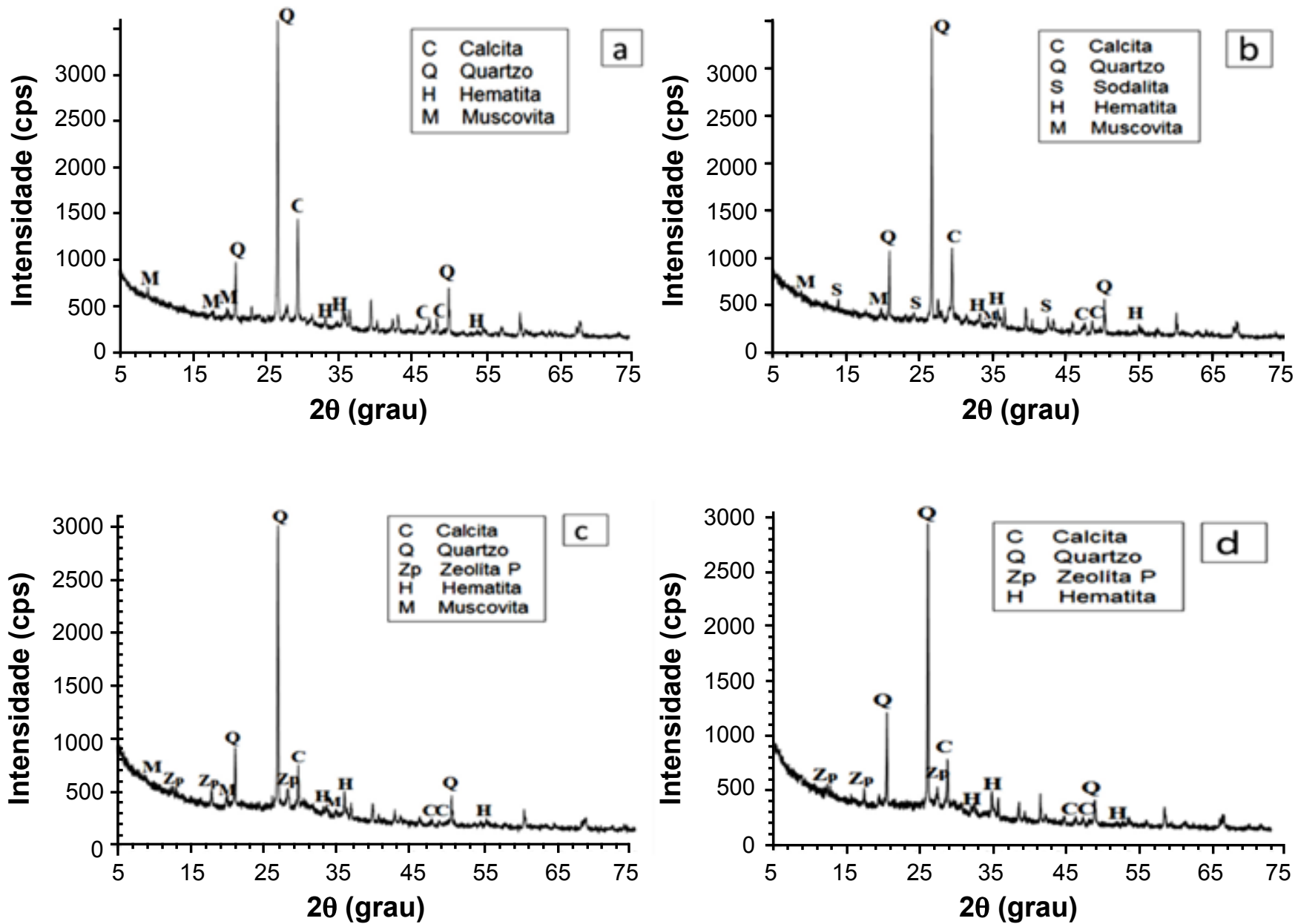

Figura 6: Difratogramas de raios $\mathrm{X}$ dos produtos zeolíticos com razões molares de $\mathrm{Si} / \mathrm{Al}$ igual a 3 a $60{ }^{\circ} \mathrm{C}$ (a) 2,12 (b) 13 (c) e 15 (d) a $100{ }^{\circ} \mathrm{C}$. [Figure 6: XRD patterns of the zeolite products with Si/Al molar ratio equal 3 at $60^{\circ} \mathrm{C}$ (a), 2.12 (b), 13 (c) and 15 (d) at $100{ }^{\circ} \mathrm{C}$.]

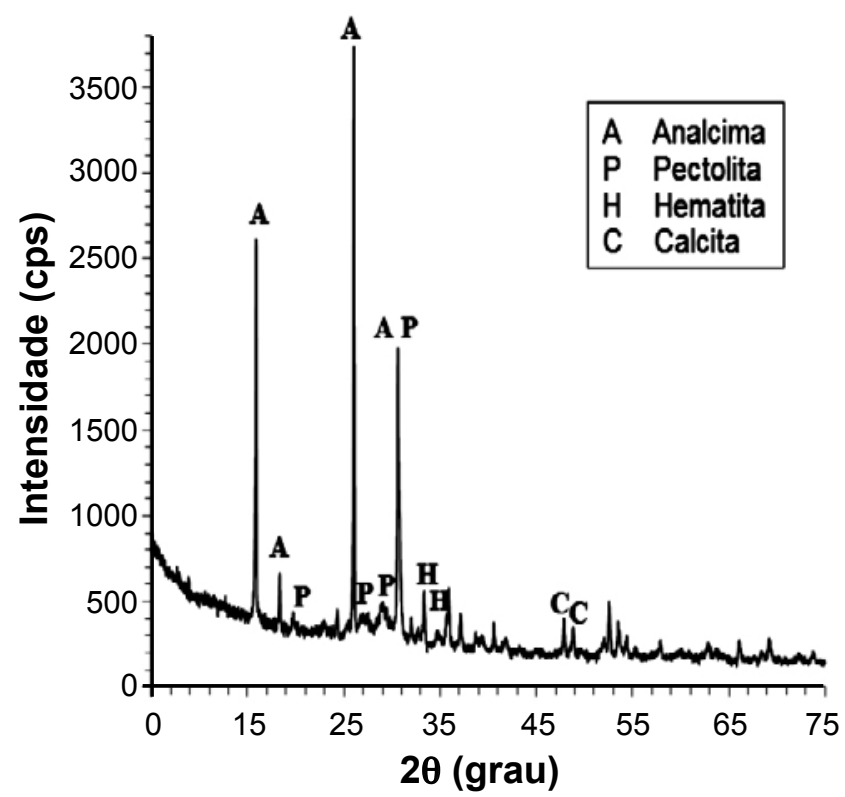

Figura 7: Difratograma de raios $\mathrm{X}$ do produto zeolítico da relação molar Si/Al igual a 7 a $190{ }^{\circ} \mathrm{C}$.

[Figure 7: XRD pattern of the zeolite synthesis product with $\mathrm{Si} / \mathrm{Al}$ molar ratio equal 7 at $190^{\circ} \mathrm{C}$.]

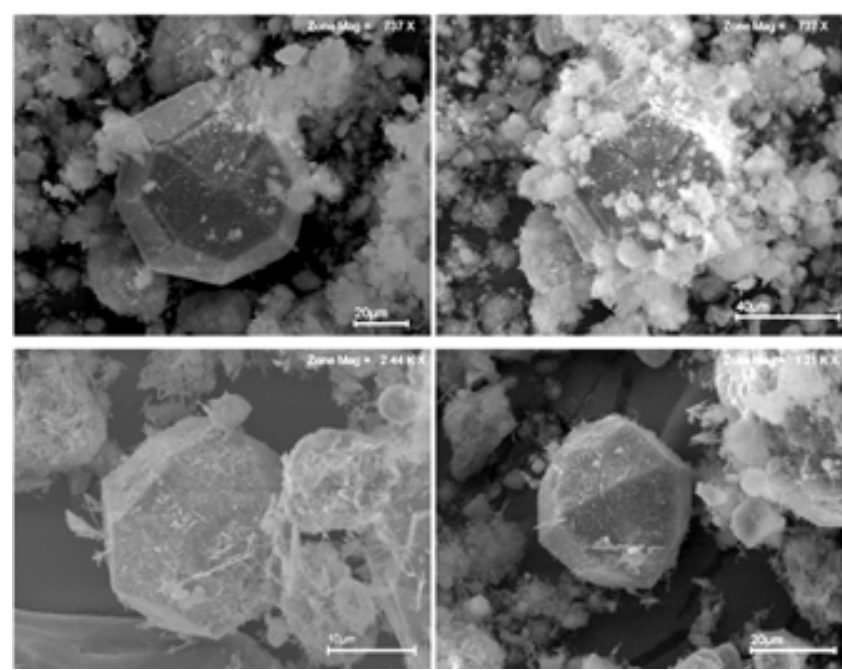

Figura 8: Fotomicrografias obtidas por microscopia eletrônica de varredura do produto zeólitico a $190{ }^{\circ} \mathrm{C}$ com relação molar de $\mathrm{Si} /$ $\mathrm{Al}$ igual a 7, apresentando cristais de pectolita em forma de agulhas sobre cristais de analcima trapezoidais.

[Figure 8: SEM micrographs of the zeolite synthesis product with Si/Al molar ratio equal 7 at $190{ }^{\circ} \mathrm{C}$, showing needles-like pectolite crystals over trapezoedric analcime crystals.] 


\section{CONCLUSÕES}

A cinza volante apresenta-se como boa fonte de silício e alumínio, para utilização como matéria prima na produção de zeólitas, pois tem em sua constituição $\sim 50 \%$ de $\mathrm{SiO}_{2}$ e $\mathrm{Al}_{2} \mathrm{O}_{3}$, o que é bastante satisfatório, uma vez que estes se configuram como elementos básicos na estrutura das zeólitas. Observa-se pela análise térmica da cinza volante a necessidade de uma calcinação prévia deste material antes de sua zeolitízação, a fim de diminuir os impactos negativos do alto teor de carbono durante este processo. Entretanto a temperatura desta calcinação deve ser bem avaliada, pois a partir de $674^{\circ} \mathrm{C}$ começa a reação de decomposição da calcita, o que irá gerar a formação de $\mathrm{CaO}$ elevando ainda mais o teor deste composto na cinza. Outra importante característica a ser avaliada é a baixa granulometria encontrada na cinza volante, tendo aproximadamente $90 \%$ de suas partículas com diâmetro abaixo de $10 \mu \mathrm{m}$, o que favorece o processo zeolítico decorrente de sua maior área de contato durante a reação de síntese. Os produtos obtidos a partir da mistura cinza/microssílica a $60^{\circ} \mathrm{C}$ não mostraram variações em suas fases mineralógicas quando comparadas com o material de partida (cinza). Foi observado somente um aumento da intensidade dos picos do quartzo em decorrência da adição de microssílica, mostrando assim que para estas condições reacionais, esta mistura não apresenta potencial zeolítico. Nas temperaturas de 150 e $190{ }^{\circ} \mathrm{C}$ produziram zeólitas com intensidades de picos mais expressivos, indicando uma maior formação das zeólitas ou maior grau de ordem cristalina destes produtos, favorecidas pela total dissolução do quartzo, gerando assim um alto teor de Si disponíveis para sua formação. A adição de microssílica à cinza foi essencial para a síntese de um maior número de fases zeolíticas, pois a utilização da cinza volante in natura possibilitou somente a formação de dois tipos de zeólitas (sodalita e tobermorita) sendo maximizado para mais três (analcima, philipsita e zeólita P) a partir desta adição. Apesar da alta concentração de metais pesados encontrados na cinza volante, estes não se configuraram como elementos prejudiciais ao processo de zeolitização, porém a adequação da solução remanescente deste processo deve ser avaliada, uma vez que se deve obedecer as principais normas referentes a descarte de rejeitos líquidos.

\section{REFERÊNCIAS}

[1] L. Reijnders, Resour. Conserv. Recycl. 43 (2005) 313.

[2] L. S. Ferret, Tese Dr., Universidade Federal do Rio Grande do Sul, RS (2004).

[3] A. M. Paprocki, R. A. Cardoso, C. M. N. Ligabue, J. A. Azevedo, F. Villwock, M. J. R. Pires, Anais $18^{\circ}$ CBECIMAT, Recife, PE (2008).

[4] M. Bruno, Diss. Mestrado, IPEN-USP, S. Paulo, SP (2008).

[5] N. Murayama, H. Yamamoto, J. Shibata, Int. J. Miner. Process 64 (2002) 1.

[6] C. F. Lin, H. C. His, Environ. Sci. Technol. 29 (1995) 1109.
[7] A. Molina, C. Poole, Miner. Eng. 17 (2004) 167.

[8] X. Querol, A. Alastuey, J. L. Fernández-Turiel, A. LópezSoler, Fuel 74 (1995) 1226.

[9] A. A. Landman, Thesis Dr. Chem., University of Pretoria (2006).

[10] C. A. F. Rocha Junior, S. C. A. Santos, C. A. G. Souza, R. S.Angélica, R. F. Neves, Anais $54^{\circ}$ Cong. Bras. Ceram., Foz do Iguaçu, PR (2010) 96.

[11] X. Querol, F. Plana, A. Alastuey, A. Lopez-Soler, A. Medinaceli, A. Valero, M. J. Domingo, E. Garcia-Rojo, Fuel 80 (2001) 857.

[12] X. Querol, A. Alastuey, A. Lopez-Soler, F. Plana, J. M. Andrés, R. Juan, P. Ferrer, C. R. Ruiz, Environ Sci. Technol. 31 (1997) 2527.

[13] D. A. Fungaro, M. Bruno, Quim. Nova 32 (2009) 955.

[14] X. Querol, N. Moreno, J. C. Umaña, A. Alastuey, E. Hernández, A. L. Soler, F. Plana, Int. J. Coal Geol. 50 (2002) 413.

[15] D. W. Breck, Zeolite Molecular Sieves, Wiley \& Sons, New York, EUA (1974).

[16] J. C. Umaña, Tese Dr, Universitat Politècnica de Catalunya, Espanha (2002).

[17] H. Höller, U. Wirsching, Fortschr. Miner. 63 (1985) 21. [18] H. Nugteren, N. Moreno, E. Sebastia, X. Querol, Int. Ash Utilization Symp., Kentucky, EUA (2001) 71.

[19] S. Rayalu, S. U. Meshram, M. Z. Hasan, J. Hazard. Mater. B77 (2000) 123.

[20] S. S. Rayalu, J. S. Udhoji, K. N. Munshi, M. Z. Hasan, J. Hazard. Mater. B88 (2001) 107.

[21] C. Amrhein, G. H. Haghnia, T. S. Kim, P. A. Mosher, R. C. Gagajena, T. Amanios, L. Torre, Environ. Sci. Technol. 30 (1996)735.

[22] C. Poole, H. Prijatama, N. M. Rice, Miner. Eng. 13 (2000) 831.

[23] P. K. Kolay, D. N. Singh, M. V. R. Murti, Fuel 80 (2001) 739.

[24] S. V. Vassilev, C. G. Vassileva, Fuel 86 (2007) 1490.

[25] Associação Brasileira de Normas Técnicas, NBR 10005 (2004).

[26] S. V. Vassilev, R. Menendez, Fuel 84 (2005) 973.

[27] N. Shigemoto, H. Hayashi, K. Miyaura, J. Mater. Sci. 28 (1993) 4781.

[28] C. D. Woolard, K. Petrus, M. Van der Horst, Water SA 26 (2000) 531.

[29] Specifications for fly ash and raw or calcined natural pozzolan for use as a mineral admixture in Portland cement ASTM - American Society for Testing and Materials, ASTM C618 (1989).

[30] Associação Brasileira de Normas Técnicas, NBR 10004 (2004).

[31] X. Querol, N. Moreno, A. Alastuey, R. Juan, J. M. Andrés, A. López-Soler, C. Ayora, A. Medinaceli, A. Valero, Geologica Acta 5 (2007) 49-57.

[32] H. Singh, P. K. Kolay, Water Air Soil Pollut. 198 (2009) 87.

[33] N. Moreno, X. Querol, J. M. Andrés, K. Stanton, M. Towler, H. Nugteren, M. Janssen-Jurkovicová, R. Jones, 
Fuel 84 (2005) 1351.

[34] X. Querol, A. Alastuey, N. Moreno, E. AlvarezAyuso, A. García-Sánchez, J. Cama, C. Ayora, M. Simón, Chemosphere 62 (2006) 171.

[35] P. K. Mehta, P. J. M. Monteiro, Concreto: Estrutura, Propriedades e Materiais, Ed. Pini, S. Paulo, SP (1994).

[36] A. M. Paprocki, M. J. R. Pires, Utilização de zeólitas sintetizadas a partir de cinzas de carvão no tratamento de efluentes de mineração e avaliação do Reuso da água, Rel. Parc. Ativ., Programa de Pós-Grad. Eng. Tecnol. Mater., PUCRS, Porto Alegre, RS (2008).
[37] Fernandes, I. D. et al. Crystalline microstructure modification of brazilian coal ash with alkaline solution, Proc. Int. Ash Utilization Symp. 3 (1999), Lexington, Kentucky, USA. (1999) 239-246.

[38] Ferret, L. et al. Gaseificação e tratamento de efluentes, in: E. C. Teixeira, (Coord.), Meio ambiente e carvão: impactos da exploração e utilização, Porto Alegre, FEPAM (2002) p.117-147. (Cadernos de planejamento e gestão ambiental, 2).

[39] A. Singer, V. Berkgaut, Environ. Sci. Technol. 29 (1995) 1748.

(Rec.13/11/2010, Rev. 21/02/2011, Ac. 19/05/2011) 